\title{
ECMO em Recém-nascidos com Hérnia Diafragmática Congénita: A Experiência de um Centro de Referência de ECMO em Portugal
}

\section{ECMO in Neonates with Congenital Diaphragmatic Hernia: The Experience of a Portuguese ECMO Referral Center}

\author{
Mariana MIRANDA $\triangle^{1,2}$, Francisco ABECASIS ${ }^{1}$, Sofia ALMEIDA ${ }^{1}$, Erica TORRES ${ }^{1}$, Leonor BOTO ${ }^{1}$, Cristina CAMILO ${ }^{1}$, \\ José Pedro NEVES ${ }^{3}$, Miguel ABECASIS ${ }^{3}$, Miroslava GONÇALVES ${ }^{4}$, Carlos MONIZ ${ }^{5}$, Marisa VIEIRA ${ }^{1}$ \\ Acta Med Port 2020 Dec;33(12):819-827 - https://doi.org/10.20344/amp.13075
}

\section{RESUMO}

Introdução: A utilização de oxigenação por membrana extracorporal (ECMO) é considerada por muitos autores como um dos mais importantes avanços tecnológicos nos cuidados de recém-nascidos com hérnia diafragmática congénita. O principal objetivo deste estudo foi reportar a experiência de um centro de oxigenação por membrana extracorporal português no tratamento de hérnia diafragmática congénita.

Material e Métodos: Estudo retrospetivo descritivo dos recém-nascidos com hérnia diafragmática congénita com necessidade de suporte de ECMO, numa unidade de Cuidados Intensivos Pediátricos de janeiro de 2012 a dezembro de 2019. Colheita de dados com recurso ao registo da Extracorporeal Life Support Organization e registo da unidade.

Resultados: Incluídos 14 recém-nascidos, todos com hérnia diafragmática congénita esquerda, num total de 15 ciclos de ECMO veno-arterial. Mediana de idade gestacional de 38 semanas e de peso ao nascer de 2,950 kg. A correção cirúrgica foi realizada antes da entrada em ECMO em seis, durante em sete e após ciclo em um caso. A mediana de idade de colocação foi de 3,3 dias e a média de duração do ciclo foi de 16 dias. Previamente à ECMO, todos os recém-nascidos apresentavam hipoxemia e acidose grave apesar de suporte ventilatório otimizado, com terapêutica com oxido nítrico e inotrópicos. Após 24 horas em ECMO, verificou-se correção de acidose, melhoria de oxigenação e estado hemodinâmico. Todos os ciclos apresentaram complicações mecânicas, sendo a mais frequente a presença de coágulos no circuito. As complicações fisiológicas mais frequentes foram as hemorrágicas e embólicas (três recém-nascidos sofreram acidente vascular cerebral isquémico durante o ciclo). Cinco crianças (35,7\%) morreram, estando todos os casos associados a complicações (duas com acidente vascular cerebral, duas com hemorragia maciça e uma descanulação acidental). A doença pulmonar crónica, má progressão ponderal e atraso do desenvolvimento psicomotor foram as morbilidades a longo prazo mais frequentes.

Discussão: Apesar dos avanços tecnológicos nos cuidados respiratórios e melhoria da segurança da técnica ECMO, o manuseamento destes recém-nascidos é complexo e existem ainda várias questões em aberto, incluindo a selecção apropriada dos doentes, a melhor abordagem e tempo de correcção cirúrgica, e o tratamento da hipertensão pulmonar na presença de shunts fetais persistentes. Conclusão: A taxa de sobrevivência foi superior à reportada no relatório da Extracorporeal Life Support Organization de 2017 (64\% vs 50\%). As complicações mecânicas e hemorrágicas foram muito prevalentes.

Palavras-chave: Hérnias Diafragmáticas Congénitas; Oxigenação por Membrana Extracorporal; Portugal; Recém-Nascido

\section{ABSTRACT}

Introduction: The use of extracorporeal membrane oxygenation (ECMO) is considered by many authors as one of the most important technological advances in the care of newborns with congenital diaphragmatic hernia. The main objective of this study was to report the experience of a Portuguese ECMO center in the treatment of congenital diaphragmatic hernia.

Material and Methods: Descriptive retrospective study of newborns with congenital diaphragmatic hernia requiring ECMO support in a Pediatric Intensive Care Unit from January 2012 to December 2019. Data collection using the Extracorporeal Life Support Organization registration and unit data base.

Results: Fourteen newborns were included, all with left congenital diaphragmatic hernia, in a total of 15 venoarterial ECMO cycles. The median gestational age was 38 weeks and the median birth weight was $2.950 \mathrm{~kg}$. Surgical repair was performed before entry into $\mathrm{ECMO}$ in six, during in seven and after in one newborn. The average age at placement was 3.3 days and the median cycle duration was 16 days. Prior to ECMO, all newborns had severe hypoxemia and acidosis despite optimized ventilatory support, with nitric oxide and inotropic therapy. After 24 hours on ECMO, there was correction of acidosis, improvement of oxygenation and hemodynamic stability. All cycles presented mechanical complications, the most frequent being the presence of clots in the circuit. The most frequent physiological complications were hemorrhagic and embolic (three newborns suffered an ischemic stroke during the cycle). Five newborns $(35.7 \%)$ died, all associated with complications (two strokes, two massive bleedings and one accidental decannulation). Chronic lung disease, poor weight gain and psychomotor developmental delay were the most frequent long-term morbidities.

Discussion: Despite technological advances in respiratory care and improved safety of the ECMO technique, the management of these newborns is complex and there are still several open questions, including the appropriate selection of patients, the best approach

1. Unidade de Cuidados Intensivos Pediátricos. Serviço de Pediatria Médica do Departamento de Pediatria. Hospital de Santa Maria. Centro Hospitalar Universitário Lisboa Norte. Lisboa. Portugal.

2. Serviço de Pediatria. Departamento da Mulher e Criança. Hospital Espírito Santo de Évora. Évora. Portugal.

3. Serviço de Cirurgia Cardiotorácica. Hospital de Santa Cruz. Centro Hospitalar Lisboa Ocidental. Lisboa. Portugal.

4. Serviço de Cirurgia Pediátrica. Departamento de Pediatria. Hospital de Santa Maria. Centro Hospitalar Universitário Lisboa Norte. Lisboa. Portugal.

5. Serviço de Neonatologia. Departamento de Pediatria. Hospital de Santa Maria. Centro Hospitalar Universitário Lisboa Norte. Lisboa. Portugal.

$\square$ Autor correspondente: Mariana Freitas Miranda. mariana_f_miranda@hotmail.com

Recebido: 23 de janeiro de 2020 - Aceite: 04 de agosto de 2020 | Copyright @ Ordem dos Médicos 2020 
and time for surgical correction, and the treatment of pulmonary hypertension in the presence of persistent fetal shunts.

Conclusion: Survival rate was higher than reported in 2017 Extracorporeal Life Support Organization report (64\% versus 50\%). Mechanical and hemorrhagic complications were very frequent.

Keywords: Extracorporeal Membrane Oxygenation; Hernias, Diaphragmatic, Congenital; Infant, Newborn; Portugal

\section{INTRODUÇÃO}

A hérnia diafragmática congénita (HDC) é uma condição complexa responsável por significativa morbilidade e mortalidade na população neonatal, com uma incidência de 1 a 3 por 10000 recém-nascidos (RNs) vivos na Europa (dados da EUROCAT Registry of Congenital Anomalies). ${ }^{1,2}$ Consiste num defeito do diafragma que permite a herniação dos órgãos abdominais para o tórax, comprometendo o normal desenvolvimento do pulmão ipsilateral e contralateral.

A mortalidade e morbilidade nos RNs com HDC está principalmente relacionada com combinação de hipoplasia pulmonar e hipertensão pulmonar, responsáveis por insuficiência respiratória grave nas primeiras horas após o nascimento. Na hipoplasia pulmonar, o processo de geração de novos vasos (vasculogénese) e ramificação progressiva dos mesmos (angiogénese) estão comprometidos, com perfusão inadequada do pulmão e impossibilidade de acomodar o débito ventricular direito (com shunt direitoesquerdo). A vascularização pulmonar anormal, hipoxemia, acidose e lesão pulmonar associada à ventilação mecânica determinam que estes RNs tenham risco aumentado de desenvolver hipertensão pulmonar persistente do RN (HPPRN), com pior prognóstico em RNs prematuros, com anomalias cromossómicas ou outras malformações. A HDC pode ainda acompanhar-se com diferentes graus de hipoplasia e disfunção do ventrículo esquerdo, normalmente transitória, com resolução após sete a 10 dias de vida. ${ }^{3-4}$

A taxa de sobrevivência pós-natal nos centros terciários melhorou lentamente, atualmente com taxas relatadas de $70 \%$ a $92 \%$, em paralelo com a alteração dos protocolos de tratamento destes $\mathrm{RNs}{ }^{5,6} \mathrm{~A}$ intervenção cirúrgica precoce nos RNs gravemente doentes foi substituída por estabilização com suporte pré-operatório intensivo, com o objetivo de evitar a lesão pulmonar, seguido da correção cirúrgica. Importantes avanços nos cuidados perinatais nas últimas décadas ajudaram na melhoria dos cuidados prestados a estes RNs, incluindo a adoção de parâmetros de ventilação mínimos e hipercapnia permissiva, a disponibilidade de óxido nítrico inalado (NO), ventilação de alta frequência oscilatória (VAFO) e oxigenação por membrana extracorporal (ECMO). ${ }^{4-6}$

Vários estudos confirmaram o beneficio do ECMO na melhoria da sobrevivência de crianças com HDC gravemente doentes, especificamente o seu papel como terapêutica de resgate e estabilização peri-operatória. ${ }^{7,8}$ Contudo é importante relembrar que o ECMO está associado a um significativo conjunto de complicações, inerentes à própria técnica, que podem causar importante morbilidade e mortalidade entre os sobreviventes. ${ }^{3}$

Existe uma lacuna de dados nacionais neste tema, com apenas quatro casos nacionais descritos. ${ }^{9,10} \mathrm{O}$ principal objetivo deste estudo foi descrever a experiência de um cen- tro Português de ECMO no tratamento de HDC, incluindo os critérios usados para iniciar ECMO, complicações reportadas, mortalidade e morbilidade a curto e longo prazo.

\section{MATERIAL E MÉTODOS}

Estudo retrospetivo descritivo com avaliação dos RNs (idade $\leq 28$ dias) com HDC com necessidade de ECMO para suporte cardiorrespiratório, na Unidade de Cuidados Intensivos Pediátricos (UCIPed), centro de referência em ECMO do Centro Hospitalar Universitário Lisboa Norte, durante um período de 8 anos (janeiro de 2012 a dezembro de 2019).

A colheita de dados foi realizada com recurso ao registo da unidade e registo ELSO (Extracorporeal Life Support Organization), organização internacional de registo de doentes que realizaram ECMO. Incluiu a colheita dos seguintes dados: características demográficas; historial médico pré e neonatal; malformações/ anomalias congénitas associadas; ventilação e terapêuticas pré-ECMO; tempo de correção cirúrgica; avaliação hemodinâmica e gasimétrica pré e em ECMO; características do ciclo de ECMO; complicações, morbilidade e mortalidade associadas ao ECMO.

Parto pré-termo foi definido como ocorrido antes ou até as 37 semanas completas de gestação e parto de termo foi definido como entre 37 semanas e as 40 semanas mais seis dias. As malformações/ anomalias congénitas pesquisadas incluíram anomalias anatómicas e cromossómicas.

A ventilação mecânica foi classificada como VAFO ou ventilação mecânica convencional. Outras variáveis pré-ECMO pesquisadas incluíram infeções documentadas, parâmetros de ventilação mecânica (mediana de pico de pressão inspiratória, de pressão positiva no final da expiração e de pressão média das vias aéreas, tipo e duração da ventilação) e gasimetria arterial $\left(\mathrm{pH}, \mathrm{PaCO}_{2}, \mathrm{PaO}_{2}\right.$ e $\left.\mathrm{SaO}_{2}\right)$. $\mathrm{O}$ índice de oxigenação, definido como a [pressão média das vias aéreas $\times$ fracção inspirada de oxigénio $\left(\mathrm{FiO}_{2}\right) \times$ $100 / \mathrm{PaO}_{2}$ ] foi calculado a partir dos dados fornecidos pelo registo ELSO.

$O$ modo do ECMO foi categorizado em veno-arterial (VA) ou veno-venoso (VV). Foi também registado o número de ciclos de ECMO em cada RN.

As complicações ocorridas durante o período em ECMO foram categorizadas como mecânicas ou fisiológicas, de acordo com as classificações do registo ELSO. As complicações mecânicas incluem: falência da membrana oxigenadora; falência da bomba; rotura dos circuitos; problemas com as cânulas (mau posicionamento, obstrução por coágulos); embolia gasosa. As complicações fisiológicas incluem: hemorragia (no território cirúrgico, local de inserção da cânula, hemopericárdio, retroperitoneu, sistema nervoso central (SNC), gastrointestinal e pulmonar); lesão renal aguda, definida como creatinina sérica superior a 
$1,5 \mathrm{mg} / \mathrm{dL}$ e/ ou necessidade de terapêutica de substituição renal (diálise ou hemodiafiltração veno-venosa contínua); lesão do SNC, incluindo convulsões (evidência clínica e/ ou eletrográfica), evidência imagiológica de lesão isquémica ou hemorrágica do SNC; desequilíbrios hidroeletrolíticos e metabólicos; complicações cardíacas, incluindo hipertensão, arritmias, isquemia, tamponamento cardíaco e uso de agentes inotrópicos/ vasopressores; e outras complicações, incluindo infeções, pneumotórax, hemólise e úlceras de pressão. Para todas as complicações registadas foram pesquisadas morbilidade e mortalidade associadas.
Foi realizada a análise de dados de seguimento de longo prazo, tendo sido considerados os RN com mínimo de um ano após fim de ciclo de ECMO. Foram consideradas como sequelas major a paralisia cerebral, cegueira, surdez, alterações profundas da compreensão e epilepsia. A existência e grau de doença pulmonar crónica foi definida de acordo com os critérios do consenso de 2001 do NICHD (National Institute of Child Health and Human Development) de displasia broncopulmonar. O diagnóstico de atraso do desenvolvimento psicomotor foi realizado por pediatra da área do Desenvolvimento infantil. A má progressão

Tabela 1 - Características da amostra de recém-nascidos com HDC em ECMO

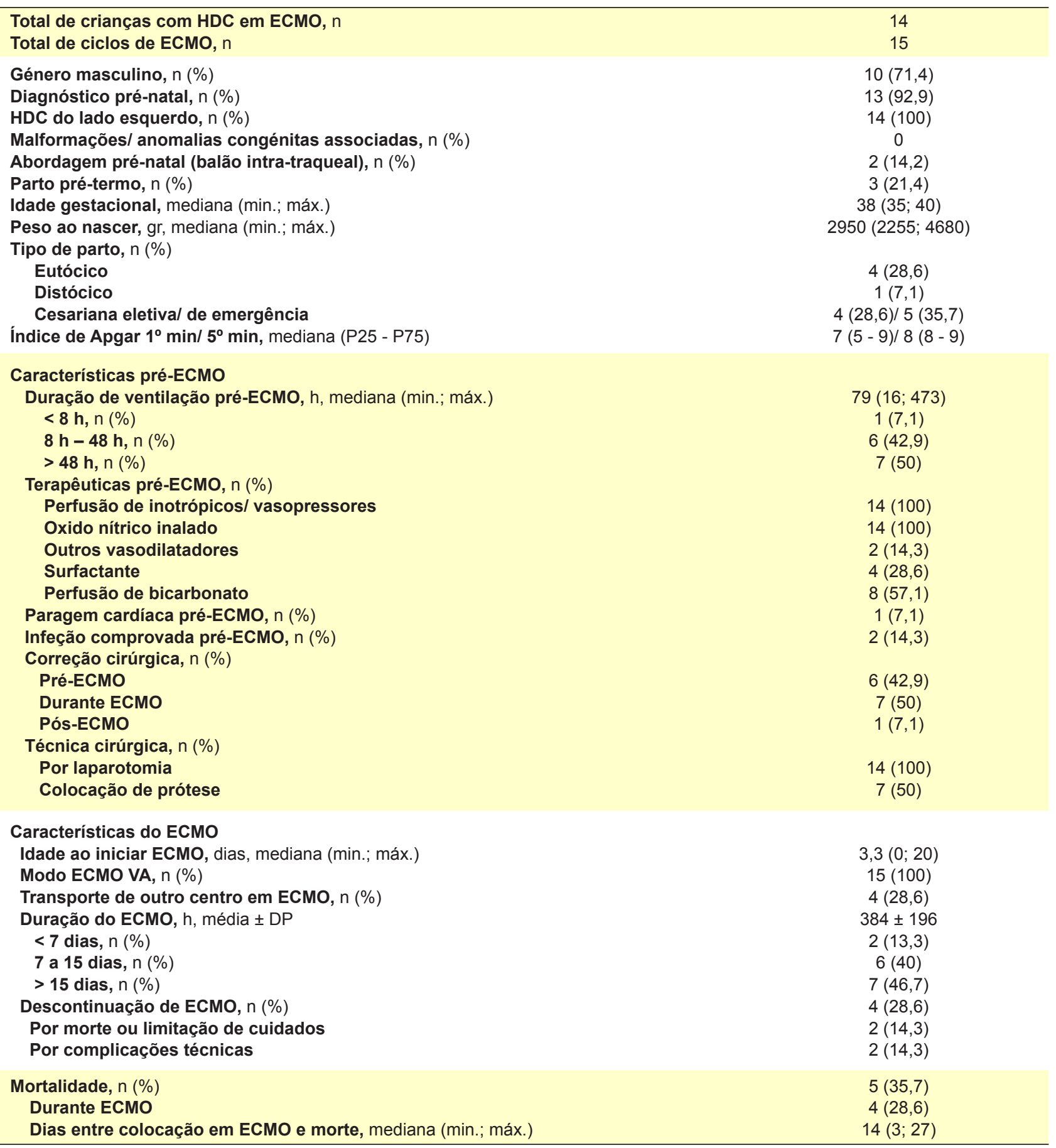

DP: desvio padrão; HDC: hérnia diafragmática congénita; ECMO: oxigenação por membrana extracorporal; VA: veno-arterial 
ponderal foi definida como peso abaixo do percentil 3 ou cruzamento de mais de dois percentis nas curvas de crescimento da Organização Mundial de Saúde de 2006, em vigor em Portugal.

Foi realizada análise estatística descritiva dos dados com uso de Microsoft Exce/ ${ }^{\circ}$. O outcome primário estudado foi a morte antes da alta hospitalar. Este estudo foi realizado de acordo com os regulamentos estabelecidos pela Comissão de Investigação Clínica e Comissão de Ética da instituição onde decorreu o mesmo. Tratando-se de um estudo observacional com confidencialidade e privacidade de dados, dada a utilização de dados de uma plataforma internacional autorizada pelo Conselho de Administração, os autores não consideraram necessário submeter este estudo a aprovação pelas referidas comissões.

\section{RESULTADOS}

\section{Amostra}

No período de oito anos, 14 crianças com HDC necessitaram de suporte cardiopulmonar em ECMO, perfazendo um total de 15 ciclos. As característica da amostra estão resumidas na Tabela 1 .

O tempo de correção cirúrgica da HDC variou, sendo que sete RNs realizaram correção durante o ciclo de ECMO, seis antes de colocação em ECMO e um após ciclo de ECMO. A técnica cirúrgica realizada foi por laparotomia em todos os casos, com colocação de prótese diafragmática em metade.
A mediana de idade dos RNs quando colocados em ECMO foi de 3,3 dias de vida. Antes do início da técnica todos os RNs tinham atingido o máximo de otimização terapêutica: apresentavam hipoxemia (mediana de índice de oxigenação de 81,8 ) e acidose grave apesar do suporte ventilatório e cardiovascular, com ventilação mecânica convencional/ VAFO com parâmetros ventilatórios agressivos e sob $100 \%$ de fração de oxigénio inspirado, oxido nítrico e terapêutica com inotrópicos (Tabelas 1 e 2). Um dos $\mathrm{RN}$ que foi colocado em ECMO tinha sofrido uma paragem cardíaca prévia, com recuperação após curto período de reanimação cardiopulmonar.

O método de ECMO utilizado em todos os RNs foi o VA com drenagem através da canulação da veia jugular interna direita com cânulas Bio-Medicus ${ }^{\circledR}$ de $8 \mathrm{~F}$ a $12 \mathrm{~F}$ e retorno através da canulação da artéria carótida primitiva direita com cânulas de 8F a 10F. A canulação foi realizada por técnica cirúrgica com desbridamento e exposição dos vasos.

A média \pm DP de duração do ECMO foi de $384 \pm 196$ horas (16 dias $\pm 8,2$ dias), com necessidade de descontinuação do ECMO em quatro: dois devido a complicações técnicas (um com descanulação acidental e um caso de falência da bomba); nos outros dois por morte do RN em ECMO ou decisão de limitação de cuidados por mau prognóstico, nomeadamente por lesões neurológicas graves.

Tabela 2 - Comparação de parâmetros pré e às 24 horas em ECMO

\begin{tabular}{|c|c|c|}
\hline & Pré-ECMO & 24h em ECMO \\
\hline \multicolumn{3}{|l|}{ Tipo de ventilação, n (\%) } \\
\hline Convencional & $1(6,7)$ & $12(80)$ \\
\hline Alta-frequência (VAFO) & $14(93,3)$ & $3(20)$ \\
\hline \multicolumn{3}{|c|}{ Parâmetros de ventilação, mediana (P25 - P75) } \\
\hline PIP, $\mathrm{cmH}_{2} \mathrm{O}$ & $27(n=1)$ & $20(20-21)(n=12)$ \\
\hline PEEP, $\mathrm{cmH}_{2} \mathrm{O}$ & $6(n=1)$ & $5(n=12)$ \\
\hline MAP, $\mathrm{cmH}_{2} \mathrm{O}$ & $19,5(18-21)(n=14)$ & $11(10-12)(n=3)$ \\
\hline Delta P & $41(38-45)(n=14)$ & $23(n=3)$ \\
\hline $\mathrm{FiO}_{2}, \%$ & 100 & 40 \\
\hline \multicolumn{3}{|c|}{ Gasimetria arterial, mediana (P25 - P75) } \\
\hline pH & $7,05(6,87-7,18)$ & $7,37(7,34-7,385)$ \\
\hline $\mathrm{PaCO}_{2}, \mathrm{mmHg}$ & $74(62,4-95,0)$ & $39(37,2-47,0)$ \\
\hline $\mathrm{PaO}_{2}, \mathrm{mmHg}$ & $25,7(19,0-35,0)(\mathbf{n}=11)$ & $78(46,5-80,0)(n=10)$ \\
\hline $\mathrm{SaO}_{2}, \%$ & $53(46,5-69,5)(n=13)$ & $98(46,5-80,0)$ \\
\hline Índice de oxigenação & $81,8(54,3-100,0)(\mathbf{n}=11)$ & $5,9(5,5-10,4)(n=10)$ \\
\hline $\mathrm{SvO}_{2}, \%$ & $41(34,5-53,5)(n=4)$ & $84(78-86)$ \\
\hline \multicolumn{3}{|c|}{ Parâmetros hemodinâmicos, mediana (P25 - P75) } \\
\hline PA sistólica, $\mathrm{mmHg}$ & $42(39,0-54,0)$ & $66(54,5-74,0)$ \\
\hline PA diastólica, $\mathrm{mmHg}$ & $30(20,5-32,0)$ & $40(34,0-53,0)$ \\
\hline PA média, $\mathrm{mmHg}$ & $35(30,0-39,0)$ & $45(42,5-59,0)$ \\
\hline
\end{tabular}

Dados relativos aos 15 ciclos, mas nem todos os dados de todos os parâmetros descritos estão disponíveis.

HDC: hérnia diafragmática congénita; ECMO: oxigenação por membrana extracorporal; PIP: pico de pressão inspiratória; PEEP: pressão expiratória final positiva; MAP: pressão média das vias aéreas; $\mathrm{FiO}_{2}$ : fração de oxigénio inspirado; $\mathrm{PaO}_{2}$ : pressão parcial de oxigénio; $\mathrm{SaO}_{2}$ : saturação de oxigénio; $\mathrm{SvO}_{2}$ : saturação venosa mista de oxigénio; $\mathrm{FiO}$ : fração de oxigénio inspirado; PA: pressão arterial 


\section{Evolução em ECMO}

A evolução clínica em ECMO, representada pela avaliação hemodinâmica e gasimétrica pré e às 24 horas em ECMO (Tabela 2), foi claramente benéfica: redução de parâmetros de ventilação para valores mínimos de repouso, observando-se uma melhoria significativa da oxigenação; correção de acidose grave; e evidente melhoria do estado hemodinâmico, com a mediana de pressão arterial média a evoluir de 35 para $45 \mathrm{mmHg}$ após 24 horas de ECMO. O débito da bomba de ECMO 24 horas após o início de ECMO variou entre 0,26 e $0,45 \mathrm{~L} / \mathrm{min}$.

\section{Complicações associadas ao ECMO}

A descrição completa das complicações reportadas durante ECMO pode ser consultado na Tabela 3.. Todos os ciclos de ECMO apresentaram complicações mecânicas, sendo a mais frequente a presença de coágulos no circuito, levando a múltiplas mudanças de circuito e remoção frequente de coágulos. As complicações fisiológicas mais frequentes foram as hemorrágicas, a infeção comprovada por cultura, o desen-

Tabela 3 - Complicações reportadas em ECMO

\begin{tabular}{lc}
\hline Mecânicas, n (\%) & \\
\hline Coágulos no circuito & $15(100,0)$ \\
Outras & $10(66,7)$ \\
Falência da bomba & $2(13,3)$ \\
Ar no circuito & $1(6,7)$ \\
Falência de oxigenador & $1(6,7)$ \\
Descanulação acidental & $1(6,7)$ \\
\hline Fisiológicas, n (\%) & \\
\hline Renais & \\
Lesão renal aguda & $1(6,7)$ \\
Terapêuticas de substituição renal & $1(6,7)$ \\
Hematológicas & \\
Coagulação intravascular disseminada & $4(26,7)$ \\
Trombólise & $1(6,7)$ \\
Hemorrágicas & \\
Hemorragia no local cirúrgico & $7(46,7)$ \\
Hemorragia no local de canulação & $5(33,3)$ \\
Gastrointestinal & $4(26,7)$ \\
Hemotórax & $2(13,3)$ \\
Outros & $2(13,3)$ \\
Pulmonar & $1(6,7)$ \\
Cardíacas & \\
Derrame pericárdico & $2(13,3)$ \\
Reanimação cardiopulmonar durante ECMO & $2(13,3)$ \\
\hline Stunning do miocárdio & $1(6,7)$ \\
Metabólicas & $1(6,7)$ \\
Hiperbilirrubinémia & $1(6,7)$ \\
Sistema nervoso central, n (\%) & \\
AVC isquémico & $3(20,0)$ \\
Hemorragia & \\
Convulsões & $3(20,0)$ \\
Outras & $2(13,3)$ \\
Infeção comprovada por cultura & $1(6,7)$ \\
Pneumotórax (com necessidade de tratamento) \\
Quilotórax & \\
\hline
\end{tabular}

ECMO: oxigenação por membrana extracorporal; AVC: acidente vascular cerebral. volvimento de pneumotórax e quilotórax. É importante ainda referir as complicações no sistema nervoso central, nomeadamente o acidente vascular cerebral isquémico que foi relatado em três $\mathrm{RNs}$, associado a alta mortalidade e morbilidade (dois destes RNs faleceram nesta sequência e o outro apresenta tetraparésia espástica sequelar).

\section{Mortalidade e morbilidade}

Verificou-se o óbito em cinco RNs, incluindo os quatro casos de necessidade de descontinuação de ECMO. A mediana de dias entre a colocação em ECMO e morte foi de 14 (intervalo entre 3 e 27). O RN que necessitou de dois ciclos de ECMO sobreviveu.

Os cinco óbitos que ocorreram estiveram todos associados a complicações hemorrágicas ou embólicas: um RN apresentou acidente vascular cerebral isquémico extenso após realização de septostomia de Rashkind emergente por dilatação das cavidades esquerdas, secundária ao fluxo do ECMO na aorta; um RN apresentou acidente vascular cerebral após um episódio de interrupção súbita do circuito, com necessidade de manobras de reanimação; um RN morreu por hemorragia cerebral, pulmonar e gastrointestinal maciça após correção cirúrgica da HDC durante ciclo de ECMO; um RN morreu por hemorragia maciça através de dreno abdominal e torácico, com necessidade de suspender o ECMO devido à impossibilidade de realizar anticoagulação; o último RN morreu por descanulação acidental após substituição de cânula arterial.

Quando comparamos o grupo de RNs sobreviventes com os não sobreviventes (Tabela 4), apesar dos grupos serem pequenos e não permitirem tratamento estatístico, é visível que o grupo não sobrevivente era constituído por RNs mais prematuros e com menor peso ao nascer.

Todas as nove crianças sobreviventes foram acompanhadas em consulta de seguimento pós-ECMO por equipa da unidade de cuidados intensivos, consulta de desenvolvimento, cirurgia pediátrica e pneumologia pediátrica, com tempo médio de seguimento de 4,8 anos (intervalo de um a oito anos). A má progressão ponderal, atraso do desenvolvimento psico-motor e o desenvolvimento de displasia broncopulmonar moderada a grave foram as morbilidades a longo-prazo mais frequentes (Tabela 5). Uma criança desenvolveu sequelas neurológicas graves e irreversíveis, com tetraparésia espástica sequelar e epilepsia.

\section{DISCUSSÃO \\ ECMO na HDC}

Os RNs com HDC frequentemente apresentam insuficiência cardiorrespiratória grave no período imediato pós-parto e primeiras semanas de vida, sendo a indicação mais comum de ECMO por insuficiência respiratória neonatal e representando cerca de $28 \%$ de todas as indicações de ECMO no período neonatal. ${ }^{7}$ Neste sentido, é lógica e fundamental a discussão e eventual referenciação pré-natal para um centro de ECMO, sobretudo nos casos com maior gravidade, de forma a evitar um transporte inter-hospitalar em ECMO, caso seja necessária esta técnica. 
Tabela 4 - Comparação das características do grupo de sobreviventes e não sobreviventes

\begin{tabular}{|c|c|c|}
\hline & Sobreviventes $(n=9)$ & Não sobreviventes $(n=5)$ \\
\hline \multicolumn{3}{|l|}{ Fatores pré-ECMO } \\
\hline Parto pré-termo, $n$ & 0 & 3 \\
\hline Idade gestacional, mediana (min.; máx.) & $39(37 ; 40)$ & $36(36 ; 39)$ \\
\hline Peso ao nascer, gr, mediana (min.; máx.) & $3050(2340 ; 4680)$ & $2725(2255 ; 3200)$ \\
\hline Duração de ventilação pré-ECMO, h, mediana (min.; máx.) & $55,5(16 ; 473)$ & $79(17 ; 219)$ \\
\hline Paragem cardíaca pré-ECMO, $\mathrm{n}$ & 1 & 0 \\
\hline Infeção comprovada pré-ECMO, n & 2 & 0 \\
\hline \multicolumn{3}{|l|}{ Correção cirúrgica, $n$} \\
\hline Pré-ЕСмо & 4 & 2 \\
\hline Durante ECMO & 4 & 3 \\
\hline Pós-ECMO & 1 & 0 \\
\hline Com prótese & 4 & 3 \\
\hline \multicolumn{3}{|l|}{ Gasimetria pré-ECMO, mediana (P25 - P75) } \\
\hline $\mathrm{pH}$ & $6,89(6,82-7,05)$ & $7,26(7,13-7,3)$ \\
\hline Índice de oxigenação & $85,5(40,0-100,9)(n=6)$ & $80,8(55,7-149,2)$ \\
\hline PA média, mmHg, mediana (P25 - P75) & $34,5(28,0-38,0)$ & $36(29,0-49,5)$ \\
\hline \multicolumn{3}{|l|}{ Fatores associados ao ECMO } \\
\hline Idade ao iniciar ECMO, dias, mediana (min.; máx.) & $2(0 ; 20)$ & $3(1 ; 9)$ \\
\hline Duração do ECMO, h, mediana (min.; máx.) & $363(75 ; 737)$ & $356(53 ; 524)$ \\
\hline Descontinuação de ECMO, n & 0 & 4 \\
\hline
\end{tabular}

HDC: hérnia diafragmática congénita; ECMO: oxigenação por membrana extracorporal; PA: pressão arterial

Embora as taxas de sobrevivência superiores a $70 \%$ sejam possíveis para as crianças com HDC, para as que requerem ECMO a taxa de sobrevivência reportada é de cerca de $50 \%$, valor que se tem mantido estável nas duas últimas décadas. A taxa de sobrevivência de RNs com HDC em ECMO na nossa população foi de $64 \%$, superior à taxa de $50 \%$ reportada no último ELSO Registry Report que avalia a HDC separadamente (dados referentes ao ano de 2017, relativos a 7,889 ciclos de ECMO de RNs com HDC). ${ }^{7}$ Esta dificuldade em melhorar a taxa de sobrevivência pode ser explicada em parte pela seleção dos RNs, visto que apenas os RNs em estado mais grave, que não são candidatos a correção cirúrgica precoce ou com hipertensão pulmonar grave, serão candidatos para ECMO. ${ }^{6,7,11,12}$

A lógica da utilização de ECMO nestes RNs deve-se a

\begin{tabular}{lc} 
Tabela 5 - Seguimento a longo prazo dos sobreviventes ( $\mathrm{n}=8$ ) \\
\hline Hipoplasia pulmonar esquerda & 8 \\
Doença pulmonar crónica & 7 \\
Má progressão ponderal & 6 \\
Atraso do desenvolvimento psico-motor & 3 \\
Paralisia cerebral & 1 \\
Perturbação do espectro do autismo & 1 \\
Perturbação de hiperatividade e défice de atenção & 1 \\
Epilepsia & 1 \\
Sobrevivência sem sequelas major & 6 \\
\hline
\end{tabular}

Dados relativos a oito dos nove sobreviventes, dado não haver tempo suficiente de seguimento num dos casos. que a reatividade vascular pulmonar e hipertensão pulmonar irão espontaneamente melhorar nas primeiras semanas de vida e, apesar da hipoplasia pulmonar permanecer, o suporte cardiopulmonar fornecido pelo ECMO pode ser fundamental até ultrapassar este período.

Contudo, a abordagem do RN com HDC em ECMO é complexa e existem ainda várias questões em aberto apesar da extensa investigação, incluindo a seleção apropriada dos RNs para ECMO, o melhor tempo de correção cirúrgica e o tratamento da hipertensão pulmonar na presença de shunts fetais persistentes. As causas de morte em ECMO mais frequentes são a hipertensão pulmonar, insuficiência respiratória ou complicações, como hemorragia ou embolia.

Vários estudos identificaram fatores de risco associados a aumento da mortalidade de RNs com HDC em ECMO, incluindo o baixo peso ao nascer, a presença de acidemia e/ ou hipoxemia refratária. As contraindicações para a realização de ECMO incluem patologias irreversíveis e pré-existentes que limitem de forma significativa a qualidade de vida, como lesões graves do sistema nervoso central. ${ }^{3-5} \mathrm{O}$ peso do $\mathrm{RN}$ e a necessidade de anticoagulação sistémica são as principais limitações técnicas, contudo a maioria das contraindicações é relativa, devendo o caso ser sempre discutido com um centro de ECMO.

Embora não existam critérios uniformemente estabelecidos para a iniciação de ECMO, a maioria das unidades considera ECMO para RNs com mais de $2 \mathrm{~kg}$ de peso e mais de 34 semanas de idade gestacional, sem outras 
Tabela 6 - Critérios para iniciar ECMO da CDH EURO Consortium Consensus de $2015^{6}$

- Incapacidade de manter saturações pré-ductais $>85 \%$ ou saturações pós-ductais $>70 \%$;

- Aumento da $\mathrm{PaCO}_{2}$ e a acidose respiratória com pH <7,15 apesar da otimização da ventilação;

- Pressão inspiratória máxima $>28 \mathrm{cmH}_{2} \mathrm{O}$ ou pressão média das vias aéreas $>17 \mathrm{cmH}_{2} \mathrm{O}$ para se conseguir atingir saturação $>$ $85 \%$;

- Entrega inadequada de oxigénio com acidose metabólica com lactato $\geq 5 \mathrm{mmol} / \mathrm{L}$ e $\mathrm{pH}<7,15$;

- Hipotensão sistémica, resistente à fluidoterapia e terapia inotrópica, com débito urinário $<0,5 \mathrm{~mL} / \mathrm{kg} / \mathrm{h}$ durante pelo menos 12 a 24 horas;

- $\quad$ Índice de oxigenação $\geq 40$ durante pelo menos 3 horas.

anomalias limitadoras de vida, após atingido o máximo de otimização do suporte ventilatório e cardiovascular. Esta unidade segue as recomendações da CDH EURO Consortium Consensus de 2015, descritas na Tabela 6.

As recomendações atuais para os RNs com HDC apoiam o conceito de minimizar o barotrauma através de limitação no pico da pressão das vias aéreas e sugerem que o início precoce de ECMO, em RNs em que esta estratégia falha, pode prevenir a lesão pulmonar. ${ }^{6,7}$

\section{Modo de ECMO para a HDC}

Apesar de historicamente a maioria das crianças com HDC ter sido tratada com ECMO veno-arterial (VA), alguns estudos sugerem que o ECMO veno-venoso (VV) pode ser igualmente eficaz. ${ }^{6,8,13}$

Muitos RNs com HDC necessitam de ECMO por compromisso cardiovascular devido a sobrecarga cardíaca direita secundária a hipertensão pulmonar grave e, em alguns casos, hipoplasia ventricular relativa com hipertensão venosa pulmonar. O ECMO VA permite diminuir o volume direcionado ao coração direito, ajudando a recuperação deste apesar da persistente elevação da resistência vascular pulmonar. No entanto, o ECMO VA está associado a manipulação e laqueação da carótida, com maior risco de complicações, particularmente hemorrágicas, o que é relevante nestes RN que podem necessitar de tratamento cirúrgico durante o ECMO. Outra possível complicação associada ao ECMO VA é o débito excessivo induzindo hipertensão arterial sistémica e disfunção ventricular esquerda grave.

O ECMO VV é tecnicamente desafiador em RNs pequenos, mas permite preservar o fluxo sanguíneo pulmonar com sangue oxigenado, o que aumenta a vasodilatação pulmonar.

Os dados sobre a associação entre o modo de ECMO e a mortalidade são inconclusivos e vários estudos mostraram resultados de sobrevida equivalentes para as duas técnicas. Contudo, o ECMO VV parece ter tendência para um perfil de risco de complicações neurológicas menor, sendo considerado por vários autores o método preferencial para RNs com HDC. No entanto, se esta técnica falhar, a conversão de ECMO VV para VA está associada a um aumento de mortalidade de $56 \%$. 8,13 Todos os RNs incluídos no nosso estudo realizaram ECMO VA, a maioria pela existência de hipertensão pulmonar grave com necessidade de suporte cardiovascular só possível pela técnica VA. Outro fator que influenciou a escolha da técnica neste grupo etário foi o facto de não existirem cânulas de duplo lúmen aramadas disponíveis no mercado Português na altura em que o programa de ECMO foi iniciado na unidade, sendo que atualmente foram retiradas do mercado a nível internacional. Sem estas cânulas não é possível fazer ECMO VV em RNs com segurança, pois a veia femoral neste grupo etário é demasiado pequena para possibilitar a canulação e as cânulas de duplo lúmen em PVC (plástico de polyvinyl chloride) podem colapsar com as bombas centrífugas utilizadas atualmente.

\section{Tempo de correção cirúrgica}

A abordagem em relação ao tempo de correção cirúrgica durante ECMO é muito variável entre centros: alguns defendem a correção precoce (< 72 horas) para permitir uma recuperação pulmonar mais precoce, enquanto outros defendem correção mais tardia, inclusive após o ciclo de ECMO, para minimizar as complicações hemorrágicas. O desenvolvimento da terapêutica anti-fibrinolítica possibilitou a diminuição do risco hemorrágico durante a correção cirúrgica em ECMO.

Vários estudos sugeriram que a sobrevida é melhor na correção em ECMO precoce e na correção pós-descanulação, contudo os estudos são inconclusivos sobre qual destas é mais vantajosa. Apesar de nos últimos anos ter-se assistido a alguma evolução na correção pós-descanulação, ainda poucos centros no mundo realizam esta técnica. ${ }^{14-16}$ Na nossa amostra apenas um RN realizou correção cirúrgica após ciclo de ECMO e apesar do bom resultado neste caso, não foi possível retirar conclusões sobre a influência do timing de correção cirúrgica na taxa de sobrevivência.

\section{Mortalidade e morbilidade de RNs com HDC em ECMO}

Os tempos médios dos ciclos de ECMO reportados aumentaram nas últimas décadas para mais de 200 horas por ciclo, em comparação com aproximadamente 150 horas na década de $1990 .{ }^{7}$ A média de duração do ciclo de ECMO na nossa amostra foi 125 horas mais longo do que a reportada no último ELSO Registry Report 2017 (384 versus 259 horas, dados referentes a crianças com HDC). ${ }^{7}$

Ciclos de ECMO mais longos e menores taxas de sobrevivência sugerem que o ECMO é cada vez mais utilizado como terapêutica de resgate em pacientes gravemente doentes e/ou com mais comorbilidades associadas. ${ }^{4}$ Os limites da duração de tratamento em ECMO ainda não estão 
estabelecidos, apesar de dados sugerirem que para além de quatro a seis semanas parece ter utilidade limitada: taxa de sobrevivência à data de alta de $56 \%$ às duas semanas de $\mathrm{ECMO}, 43 \%$ às quatro semanas, $15 \%$ às cinco semanas e após os 40 dias de ECMO não houve sobreviventes. ${ }^{4,17}$

A HDC do lado direito ocorre em cerca de $25 \%$ de todos os casos e está associada a um aumento da gravidade da hipoplasia pulmonar, com maior necessidade de terapêutica vasodilatadora pulmonar e traqueostomia. ${ }^{18} \mathrm{Na}$ nossa amostra não tivemos nenhum caso de HDC do lado direito.

Como já referido, o ECMO está associado a risco de complicações e morbilidade significativas, inerentes à própria técnica. As complicações mecânicas e hemorrágicas foram muito prevalentes na nossa amostra e importantes fatores contribuidores para os desfechos desfavoráveis. Ao longo do tempo, estas complicações levaram a algumas mudanças na estratégia de anti-coagulação. Ao longo do tempo, estas complicações levaram a algumas mudanças na estratégia de anti-coagulação, incluindo o uso de anti-agregação com ácido acetil-salicílico na ausência de hemorragia, doseamento e correção dos níveis de anti-trombina III e desenvolvimento de técnicas para remoção de coágulos das cânulas sem necessidade de substituição das mesmas.

A incidência de doença pulmonar crónica ou displasia broncopulmonar em sobreviventes de HDC reportada é de $33 \%$ a $52 \%$. Contudo, estudos mostram que nos RNs que realizaram ECMO esse valor pode aumentar em cerca de nove vezes. ${ }^{17} \mathrm{Na}$ nossa amostra, a doença pulmonar crónica moderada a grave com necessidade de oxigenoterapia prolongada foi bastante prevalente, afetando sete das oito crianças sobreviventes com tempo suficiente de seguimento $(87 \%)$.

O risco de sequelas neurológicas é maior nos sobreviventes que necessitaram de ECMO, incluindo perda auditiva neurossensorial, atraso do desenvolvimento psico-motor e perturbação do espectro do autismo. A utilização de ECMO está também associada a um risco 17 vezes maior de má progressão ponderal aos 12 meses de vida. ${ }^{19}$ Todas estas possíveis morbilidades tornam muito importante o seguimento em consulta destas crianças até à adolescência.

\section{Limitações do estudo}

As limitações deste estudo incluem ser retrospectivo, unicêntrico, baseado numa amostra pequena, com dados limitados, visto serem obtidos por consulta de base de dados internacional, e com limitado tempo de seguimento em alguns dos doentes mais recentes.

\section{CONCLUSÃO}

Apesar dos avanços tecnológicos nos cuidados respiratórios dos RNs e da melhoria da segurança do ECMO, as taxas de sobrevivência de RNs com HDC continuam baixas. A utilização da ECMO permite a sobrevivência em casos mais graves, mas não é isenta de riscos: em cerca de um terço a metade dos casos não evita a morte e está associada a significativo risco de morbilidade pulmonar e neurológica nos sobreviventes. As variações entre centros refletem muitas vezes diferentes critérios na seleção dos RNs com HDC que recebem suporte de ECMO.

No nosso estudo, a taxa de sobrevivência de RNs com HDC em ECMO foi superior à média dos registos internacionais. Apesar de este estudo apresentar limitações importantes, permite conhecer a realidade de um centro de referência do nosso país. Pretende-se também uma maior sensibilização dos obstetras, neonatologistas e cirurgiões pediátricos para a importância da discussão e referenciação atempada dos casos de HDC grave para um centro de referência de ECMO.

\section{AGRADECIMENTOS}

Os autores gostariam de agradecer a todos os profissionais envolvidos nos cuidados destes RNs em ECMO e às famílias que confiaram na equipa para implementar uma técnica pioneira no país.

\section{PROTEÇÃO DE PESSOAS E ANIMAIS}

Os autores declaram que os procedimentos seguidos estavam de acordo com os regulamentos estabelecidos pelos responsáveis da Comissão de Investigação Clínica e Ética e de acordo com a Declaração de Helsínquia da Associação Médica Mundial publicada em 2013.

\section{CONFIDENCIALIDADE DOS DADOS}

Os autores declaram ter seguido os protocolos do seu centro de trabalho acerca da publicação de dados.

\section{CONFLITO DE INTERESSES}

Os autores declaram a inexistência de conflitos de interesse na realização do presente trabalho.

\section{FONTES DE FINANCIAMENTO}

Não existiram fontes externas de financiamento para a realização deste artigo.

\section{REFERÊNCIAS}

1. European Platform on Rare Disease Registration. [consultado 2019 ago 23]. Disponível em: https://eu-rd-platform.jrc. ec.europa.eu/eurocat/ eurocat-data/prevalence.

2. McGivern M, Best K, Rankin J, Wellesley D, Greenlees R, Addor M-C, et al. Epidemiology of congenital diaphragmatic hernia in Europe: a register-based study. Arch Dis Child Fetal Neonatal Ed. 2015;100:F13744.

3. Van den Hout L, Reiss I, Felix J, Hop WC, Lally P, Lally K, et al. Risk factors for chronic lung disease and mortality in newborns with congenital diaphragmatic hernia. Neonatology. 2010;98:370-80.

4. Turek J, Nellis J, Sherwood B, Kotagal M, Mesher A, Thiagarajan RR, et al. Shifting risks and conflicting outcomes-ECMO for neonates with congenital diaphragmatic hernia in the modern era. J Pediatr. 2017;190:163-8.

5. Jancelewicz T, Brindle M, Harting M, Tolley E, Langham Jr. M, Lally P, et al. On behalf of the Congenital Diaphragmatic Hernia Study Group. Extracorporeal membrane oxygenation (ECMO) risk stratification in newborns with congenital diaphragmatic hernia $(\mathrm{CDH})$. J Pediatr Surg. 
2018;53:1890-5

6. Snoek K, Reiss I, Greenough A, Capolupo I, Urlesberger B, Wessel L, et al. Standardized postnatal management of infants with congenital diaphragmatic hernia in Europe: The CDH EURO Consortium Consensus - 2015 Update. Neonatology. 2016;110:66-74.

7. International Summary of ELSO Registry Report. Extracorporeal life support organization (ELSO). January, 2017. [consultado 2019 ago 23]. Disponivel em: http://www.elso.org/registry.

8. Delaplain P, Jancelewicz T, Nardo M, Zhang L, Yu P, Cleary J, et al. Study by ELSO CDH Interest Group. Management preferences in ECMO mode for congenital diaphragmatic hernia. J Pediatr Surg. 2019;54:903-8.

9. Marques R, Pereira S, Abecasis F, Valente S, Neves J, Abecasis M, et al. Uso de oxigenação extra corporal (ECMO) em recém-nascidos com hernia diafragmática congénita - dois casos de sucesso. Acta Pediatr Port. 2013;44:172-5.

10. Rocha G, Soares P, Henriques-Coelho T, Correia-Pinto J, Monteiro J, Guimarães $\mathrm{H}$, et al. Neonatal extracorporeal membrane oxygenation: Initial experience of Hospital de São João. Rev Port Pneumol. 2014;20:336-40.

11. Golden J, Jones N, Zagory J, Castle S, Bliss D. Outcomes of congenital diaphragmatic hernia repair on extracorporeal life support. Pediatr Surg Int. 2017;33:125-31.

12. Murthy K, Pallotto E, Gien J, Brozanski B, Porta N, Zaniletti I, et al. Predicting death or extended length of stay in infants with congenital diaphragmatic hernia. J Perinatol. 2016;36:654-9.
13. Guner Y, Khemani R, Qureshi F, Wee C, Austin M, Dorey F, et al Outcome analysis of neonates with congenital diaphragmatic hernia treated with venovenous vs venoarterial extracorporeal membrane oxygenation. J Pediatr Surg. 2009;44:1691-701.

14. Robertson J, Criss C, Hsieh L, Matsuko N, Gish J, Rodrigo A, Mon R, et al. Comparison of early versus delayed strategies for repair of congenital diaphragmatic hernia on extracorporeal membrane oxygenation. J Pediatr Surg. 2018;53:629-34.

15. Partridge E, Peranteau W, Rintoul N, Herkert L, Flake A, Adzick N, et al. Timing of repair of congenital diaphragmatic hernia in patients supported by extracorporeal membrane oxygenation (ECMO). J Pediatr Surg. 2015;50:260-2.

16. Glenn I, Abdulhai S, Lally P, Schlager A, for the Congenital Diaphragmatic Hernia Study Group. Early CDH repair on ECMO: improved survival but no decrease in ECMO duration (A CDH Study Group Investigation). J Pediatr Surg. 2019;54:2038-43.

17. Wynn J, Krishnan U, Aspelund G, Zhang Y, Duong J, Stolar C, et al. Outcomes of congenital diaphragmatic hernia in the modern era of management. J Pediatr. 2013;163:114-9.

18. Partridge E, Peranteau W, Herkert L, Rendon N, Smith H, Rintoul $\mathrm{N}$, et al. Right- versus left-sided congenital diaphragmatic hernia: a comparative outcomes analysis. J Pediatr Surg. 2016;51:900-2.

19. Danzer E, Hoffman C, D'Agostino J, Gerdes M, Bernbaum J, Antiel R, et al. Neurodevelopmental outcomes at 5 years of age in congenital diaphragmatic hernia. J Pediatr Surg. 2017;3;52:437-43. 